\title{
Paracetamol absorption test to detect poor enteric absorption of oseltamivir in intensive care unit patients with severe influenza: a pilot study
}

\author{
Faten May ${ }^{1,2}$, Gilles Peytavin ${ }^{3}$, Slim Fourati ${ }^{4}$, Claire Pressiat ${ }^{5}$, Guillaume Carteaux ${ }^{1,2}$, Keyvan Razazi ${ }^{1,2}$, \\ Armand Mekontso Dessap ${ }^{1,2}$ and Nicolas de Prost ${ }^{1,2^{*}}$ (D)
}

@ 2019 Springer-Verlag GmbH Germany, part of Springer Nature

Dear Editor,

Pandemic and seasonal influenza epidemics can be associated with a high degree of morbidity and mortality, especially in patients developing severe influenza pneumonitis with the acute respiratory distress syndrome (ARDS) or the less frequent fulminant myocarditis. Early administration of the neuraminidase inhibitor oseltamivir has been shown to reduce the duration of respiratory illness for patients infected with both influenza A and B viruses [1] and was associated with a reduction in hospital mortality in patients with severe influenza. Since the 2009 influenza pandemics, early oseltamivir administration, which can only be given orally (or through a nasogastric tube), is thus recommended by the World Health Organization in patients hospitalized for severe influenza, including those requiring intensive care (ICU) admission. Indeed, pharmacokinetic data obtained in healthy volunteers, as well as in critically ill patients [2], showed that oseltamivir phosphate (OP), the inactive prodrug, is well absorbed and rapidly converted by intestinal and hepatic esterases to the active metabolite oseltamivir carboxylate $(\mathrm{OC})$, which is renally eliminated. However, enteric absorption can be compromised in critically ill patients [3] due to impaired gut function, particularly in patients with cardiovascular failure. Unfortunately,

\footnotetext{
*Correspondence: nicolas.de-prost@aphp.fr

${ }^{1}$ Service de Réanimation Médicale, Assistance Publique-Hôpitaux de Paris, Hôpitaux Universitaires Henri Mondor-Albert Chenevier, 51, Avenue du Maréchal de Lattre de Tassigny, 94010 Créteil, France

Full author information is available at the end of the article
}

plasma OC measurements are not routinely performed in most hospitals, precluding close monitoring of OC pharmacokinetics. Using the paracetamol absorption test (PAT), Jahns et al. [4] recently demonstrated enteral drug malabsorption in two patients with fulminant influenza myocarditis and cardiogenic shock, prompting a switch to parenteral therapy. The current pilot study aimed at assessing the diagnostic performances of the PAT for detecting oseltamivir absorption failure in critically ill patients with severe influenza. The study reported on a prospective cohort of 15 patients admitted to the ICU for severe influenza during the 2018/2019 influenza epidemics. Oseltamivir was routinely prescribed at a standard dose of $75 \mathrm{mg}$ twice daily (an oral suspension of oseltamivir $6 \mathrm{mg} / \mathrm{mL}$ was given orally or through a nasogastric tube, which was then flushed with water) within the first $24 \mathrm{~h}$ and then adjusted to creatinine clearance when it was $<60 \mathrm{~mL} / \mathrm{min}$. A PAT was performed in all patients within $72 \mathrm{~h}$ of ICU admission, consisting in the measurement of plasma paracetamol concentration (homogeneous immunoassay with a limit of quantification (LOQ) of $5 \mathrm{mg} / \mathrm{L}) 60 \mathrm{~min}$ after enteral loading with $1000 \mathrm{mg}$ of paracetamol $[2,3]$. The PAT was considered positive for a plasma paracetamol concentration $\geq 10 \mathrm{mg} / \mathrm{L}$ [4]. The pharmacokinetics of oseltamivir and its metabolites were subsequently explored by measuring $\mathrm{OP}$ and $\mathrm{OC}$ trough plasma concentrations, using ultra-performance liquid chromatography with tandem mass spectrometry with $\mathrm{LOQ}<1 \mathrm{ng} / \mathrm{mL}$ and $<10 \mathrm{ng} / \mathrm{mL}$, respectively [5]. Based on pharmacokinetics data obtained in healthy subjects loaded orally with $75 \mathrm{mg}$ of oseltamivir bi-daily,

\section{Springer}


adequate $\mathrm{OC}$ trough plasma concentration was $158 \mathrm{ng} /$ $\mathrm{mL}$ (i.e., approximately $35 \%$ of the maximal concentration value) [5]. Fifteen patients (12 males/three females), aged $62 \pm 17$ years, were included (Table E1, online supplement). Among them, seven (47\%) required vasopressors, eight (53\%) invasive mechanical ventilation and four (27\%) extracorporeal membrane oxygenation support. All patients had influenza A infection except one patient who had an influenza B virus infection. Seven patients fulfilled the criteria for the ARDS, and four patients had myocarditis. Two patients died in the hospital. Eleven patients $(73 \%)$ had a positive PAT (median [range] plasma concentration of paracetamol was $13 \mathrm{mg} / \mathrm{L}$ [10$36]$ ), and all had OC trough plasma concentrations above the predefined threshold value $(651 \mathrm{ng} / \mathrm{mL}$ [201-3789]), corresponding thus to a positive predictive value of the PAT of $100 \%$ for detecting sufficient oseltamivir absorption. Among four patients who had a negative PAT, two patients-who were under extracorporeal membrane oxygenation support (ECMO) - showed undetectable OC trough plasma concentrations $(<10 \mathrm{ng} / \mathrm{mL})$, while two others had concentrations above the predefined adequate concentrations. Overall, patients having a negative PAT had significantly lower OC plasma trough concentrations than others (Fig. 1) and there was a significant and strong correlation between plasma concentrations of paracetamol measured at $60 \mathrm{~min}$ of enteral loading and oseltamivir plasma trough concentrations (Spearman's $r=0.66, p=0.009$ ). Two patients with negative PAT were switched to intravenous zanamivir.

Our data suggest that the PAT can be useful for early detection of enteric absorption failure in patients with severe influenza under oral oseltamivir treatment, particularly in those under ECMO. Until confirmatory studies are done, we suggest performing a PAT when therapeutic drug monitoring of oseltamivir is not available. If negative, switching to parenteral zanamivir therapy should be considered.

Electronic supplementary material

The online version of this article (https://doi.org/10.1007/s00134-019-05693-z) contains supplementary material, which is available to authorized users.

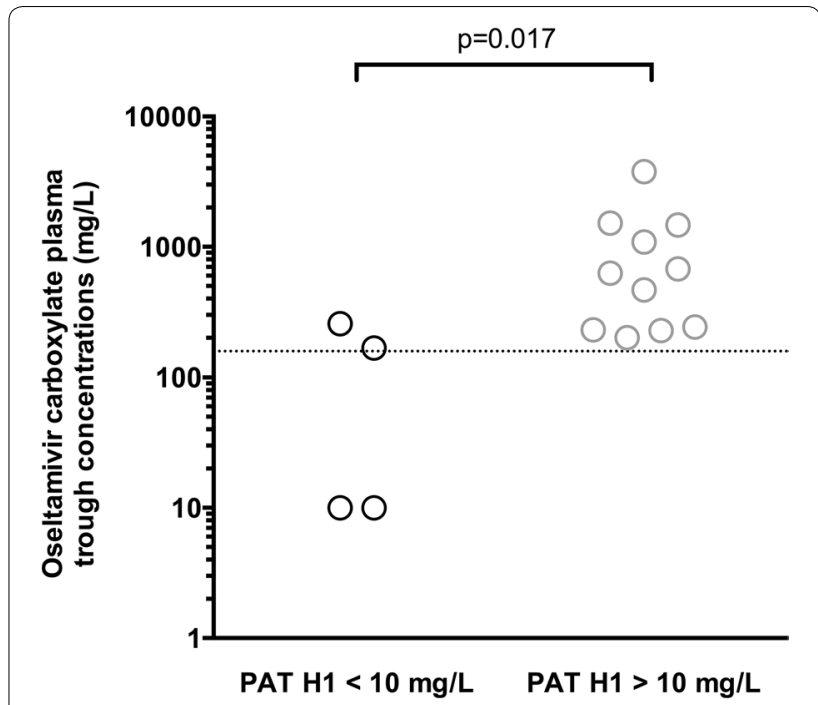

Fig. 1 Oseltamivir carboxylate trough plasma concentration in patients with positive or negative paracetamol absorption test. The dotted line indicates the predefined oseltamivir carboxylate trough concentration threshold value (i.e., $158 \mathrm{ng} / \mathrm{mL}$ ); note that the $y$-axis is a logarithmic scale; OC oseltamivir carboxylate, PAT paracetamol absorption test; the $p$ value comes from the Mann-Whitney test

Funding

None.

Compliance with ethical standards

Conflicts of interest

The authors declare that they have no conflicts of interest.

Ethical approval

All patients received information that the data abstracted from their medical charts would be used for research purposes. This study has been performed in accordance with the ethical standards laid down in the 1964 Declaration of Helsinki and its later amendments. Data were anonymized and compiled according to the requirements of the Commission Nationale Informatique et Liberté.

\section{Publisher's Note}

Springer Nature remains neutral with regard to jurisdictional claims in published maps and institutional affiliations.

Accepted: 11 July 2019

Published online: 22 July 2019

\footnotetext{
Author details

1 Service de Réanimation Médicale, Assistance Publique-Hôpitaux de Paris, Hôpitaux Universitaires Henri Mondor-Albert Chenevier, 51, Avenue du Maréchal de Lattre de Tassigny, 94010 Créteil, France. ${ }^{2}$ Groupe de Recherche Clinique CARMAS, Université Paris-Est Créteil, IMRB, Créteil, France. ${ }^{3}$ Département de Pharmaco-Toxicologie, Hôpital Bichat-Claude Bernard, Assistance Publique-Hôpitaux de Paris et IAME, UMR 1137, Université Paris Diderot, Sorbonne Paris Cité and INSERM, Paris, France. ${ }^{4}$ Département de Microbiologie, Assistance Publique-Hôpitaux de Paris, Hôpitaux Universitaires Henri Mondor-Albert Chenevier, Créteil, France. ${ }^{5}$ Laboratoire de Pharmacologie-Toxicologie, Assistance Publique-Hôpitaux de Paris, Hôpitaux Universitaires Henri Mondor-Albert Chenevier, Créteil, France.
}

References

1. Treanor JJ, Hayden FG, Vrooman PS, Barbarash R, Bettis R, Riff D et al (2000) Efficacy and safety of the oral neuraminidase inhibitor oseltamivir in treating acute influenza: a randomized controlled trial. US Oral Neuraminidase Study Group. JAMA 283(8):1016-1024

2. Ariano RE, Sitar DS, Zelenitsky SA, Zarychanski R, Pisipati A, Ahern S et al (2010) Enteric absorption and pharmacokinetics of oseltamivir in critically ill patients with pandemic (H1N1) influenza. CMAJ 182(4):357-363

3. Heyland DK, Tougas G, King D, Cook DJ (1996) Impaired gastric emptying in mechanically ventilated, critically ill patients. Intensive Care Med 22(12):1339-1344 
4. Jahns FP, Ben-Hamouda N, Kirsch M, Roumy A, Liaudet L (2018) Intravenous zanamivir for influenza myocarditis and enteral malabsorption. Crit Care 22(1):332

5. Hu ZY, Laizure SC, Meibohm B, Herring VL, Parker RB (2013) Simple and sensitive assay for quantification of oseltamivir and its active metabolite oseltamivir carboxylate in human plasma using high-performance liquid chromatography coupled with electrospray ionization tandem mass spectrometry: improved applicability to pharmacokinetic study. J Pharm Biomed Anal 72:245-250 